\title{
COMMENTARY
}

\section{"Would some form of accreditation of academic programs be beneficial to the geosciences?"}

That profound question has been under debate in our profession for some time and in many ways. Several different professional societies have considered accreditation, and multiple surveys have been conducted. Currently, GSA has an ad hoc committee studying the question. The committee was appointed to follow up on discussions that began in the meeting of the GSA Associated and Allied Societies in February 2004; discussions have continued in subsequent meetings of that and other groups within GSA

In September 2006, as a prelude to the first meeting of the ad hoc committee on accreditation, GSA conducted a survey of academic department chairs. The survey asked the chairs to identify pros and cons, and concluded with a direct question, "Are you in favor of some type of accreditation for academic programs in the geosciences?" The number of responses to the survey was an unusually high 34\% (225 returned of 662 distributed), and the responses to the direct question were 51\% (115) "no" and 49\% (110) "yes." Sorting the responses by department size (number of graduating majors per year) shows that the proportion of yes responses is inversely related to department size (the smaller departments responded $60 \%$ "yes" and 40\% "no").

The following is a listing of pros and cons derived from the survey, from volunteered correspondence, and from committee discussions:

The primary points in favor of accreditation say that it may:

- $\quad$ enhance professional recognition of the geosciences;

- $\quad$ assist departments in obtaining resources;

- $\quad$ strengthen the geosciences (as the American Chemical Society strengthens chemistry);

- $\quad$ enhance the role of the geosciences in K-12 education;

- $\quad$ identify a common knowledge base for the geosciences;

- $\quad$ establish standards for curriculum content; and

- $\quad$ provide a basis for assessment tools.

The primary points opposing accreditation say that it may:

- be too restrictive and too controlling of curriculum design in individual departments;

- require excessive time and energy to establish and administer;

- $\quad$ stifle innovation in our rapidly evolving science;

- leave some subdisciplines out;

- $\quad$ restrict the number and diversity of students; and

- lead to the closure of small departments.
We have begun a healthy debate, one that is a vital component of our overall concern for the future of the geosciences. We are a growing science: in the quality of our science, in the numbers of professional practitioners, in the diversity of subdisciplines, in innovative and interdisciplinary research, and in the practical applicability of our results. Yet we remain concerned about the degree of public and political recognition we receive, as manifest in our dissatisfaction with our professional stature relative to other fields, in levels of funding for our research, in the use of our expertise in public policy decisions, and in levels of salaries for our graduates. A greater unity and sense of common purpose will improve our status. The immediate question before us is whether some form of academic accreditation will help move the geosciences toward the corporate goals to which we all aspire.

The ad hoc committee will continue to explore the general question. For example, we are studying the system of accreditation that is already well established in the UK. Our previously circulated survey referred to accreditation only in a generic way; however, the responses commonly addressed assumed specifics. As a follow-up, we will prepare a straw program, incorporating the features that are viewed favorably and eliminating features that are viewed unfavorably. We will conduct another survey with the specifics of the straw program to obtain feedback. We already have received the comment, "If it ain't broke, don't fix it." There are many indications that our current status may not be exactly "broke," but on the other hand, few would argue that we have everything we would like to have as a science and a profession. We all agree that it is our obligation to provide the best opportunity for the next generation of geoscientists; now our responsibility is to strive to find the best way to do that. Clearly, we can do better than the status quo; to be workable, any solution must have widespread support and collaboration. We solicit your comments and concrete suggestions*. Opinions that this is a great idea or that it would be a disaster are not particularly helpful; we need explanations of how and why something might work or might not work. From those explanations, we can work toward ultimate solutions.

For the committee, Bill Thomas, chair

Committee members: Michael Arthur, Jack Sharp, Randy Keller, Chris Hepburn, John Anderson, Darrel Schmitz, Ira Sasowsky, Robert Eves, Joan Fryxell, Ed Roy, Mary Beth Gray, David Best, Edmund Nickless, Duane A. Eversoll, Jack Hess

*Go to www.geosociety.org/aboutus/accreditation.htm. 